\title{
Pain control by melatonin: Physiological and pharmacological effects (Review)
}

\author{
WEI-WEI CHEN, XIA ZHANG and WEN-JUAN HUANG \\ Department of Neurology, Xuzhou Central Hospital, Xuzhou, Jiangsu 221009, P.R. China
}

Received April 4, 2016; Accepted July 25, 2016

DOI: 10.3892/etm.2016.3565

\begin{abstract}
Pain and anxiety are the most common neurological responses to many harmful or noxious stimuli and their management clinically is often challenging. Many of the frequently used morphine-based drugs, non-steroid anti-inflammatory drugs and acetaminophen, while efficient for treating pain, lead to patients suffering from several unwanted side effects. Melatonin, produced from the pineal body is a hormone of darkness, is involved in the control of circadian rhythms, and exerts a number of pharmacological effects. Melatonin mediates its actions through MT1/MT2 melatonin receptors on the cell membrane and also through RZR/ROR nuclear orphan receptors. Chronic pain syndromes are often associated with the desynchronization of circadian and biological rhythms, which also cause disturbances in the sleep-wake cycle. Melatonin-mediated analgesic effects seem to involve $\beta$-endorphins, GABA receptor, opioid receptors and the nitric oxide-arginine pathway. The effectiveness of melatonin as an analgesic and anxiolytic agent has been demonstrated in various animal models of pain and this led to the use of melatonin clinically in different pathological conditions and also in patients undergoing surgery. Melatonin was found to be effective in many of these cases as an anxiolytic and analgesic agent, indicating its clinical application.
\end{abstract}

\section{Contents}

1. Introduction

2. Biochemistry and physiology of melatonin

3. Melatonin and pain

4. Pharmacological use of melatonin in humans

5. Melatonin and migraine

Correspondence to: Dr Wen-Juan Huang, Department of Neurology, Xuzhou Central Hospital, 199 Jiefang South Road, Xuzhou, Jiangsu 221009, P.R. China

e-mail: lrwnwe97@163.com

Key words: melatonin, circadian rhythms, $\beta$-endorphins, GABA receptor, opioid receptors
6. Melatonin and fibromyalgia

7. Irritable bowel syndrome and melatonin

8. Melatonin in the control of peri-operative outcomes

9. Conclusions

\section{Introduction}

The most common response to physically harmful or noxious stimuli is the sensation of pain, which medical practitioners have to manage in their patients and often, its management clinically is challenging. Many available and frequently used drugs based on morphine, non-steroid anti-inflammatory drugs (NSAIDs) and acetaminophen are efficient for treating pain, albeit they exhibit unwanted side effects $(1,2)$. Thus, morphine-based drugs, besides leading to addiction and dependence, also cause constipation, confusion, sleep disturbances and respiratory depression (3). Gastrointestinal (GI) damage in the form of ulcers and inflammation of stomach and duodenum is clinically evident with the use of NSAIDs (4). Chronic pain is often non-responsive to conventional medical interventions and induces anxiety and fear, which further aggravate the pain. It is well accepted that pain and anxiety positively interact with each other (5). Earlier studies on pain focused on identification of the components of peripheral pain mediators such as proteins/receptors (nociceptors) and the gate control mechanisms of pain transmission at the spinal cord (6). On the other hand, anxiety-related issues have been addressed predominantly from a psychological perspective (7). Recent advances in imaging technologies were useful in improving our understanding of the brain network(s) that may mediate pain-anxiety interaction. However, many molecular players that are involved in pain-anxiety regulation and their interactions under physiological and pathological conditions are yet to be identified. These studies may unravel better approaches to address the patients' pain and to prevent anxiety. Considering the many side effects of existing drugs, it is important to identify alternative analgesics without the unwanted side effects and to better understand the normal physiological and natural defence mechanisms against pain, which may be harnessed to clinically control pain (8).

Chronic pain syndromes are often associated with desynchronization of circadian and biological rhythms. Circadian rhythms are known to be involved in many physiological processes including the sleep-wake cycle, secretion of 
hormones, body temperature regulation and gastro-intestinal tract function $(9,10)$. Disturbances in the regulation of the circadian rhythm can lead to several pathological conditions, which themselves are shown to de-regulate circadian rhythms. Central control of biological rhythms is orchestrated by the suprachiasmatic nuclei (SCN) of the hypothalamus and certain SCN areas receive photo-signals directly via an optic nerve from the retina (11). Epiphysis or the pineal gland in the diencephalon area is above the superior colliculus of the midbrain and functions as an endocrine gland, synthesizing and secreting melatonin (N-acetyl-5-methoxytryptamine), an important neurohormone. Almost $80 \%$ of melatonin is secreted by epiphysis and other tissues such as GI tract, lungs, and renal cortex, while eye retina secrete the remaining melatonin $(12,13)$. Melatonin is considered hormone of darkness as its secretion is highest from the pineal gland during dark periods of the night and it is also referred as 'the chemical code of darkness' (14). Melatonin has been suggested to play an important role in the regulation of pain under normal physiological conditions since both pain perception as well as melatonin secretion are circadian in nature (15). In addition, melatonin has been shown to influence pain perception (16).

\section{Biochemistry and physiology of melatonin}

Melatonin biosynthesis begins with the conversion of tryptophan to serotonin, which is acetylated by arylalkylamine-N-acetyltransferase to form $\mathrm{N}$-acetyl-serotonin, followed by methylation at the 5-hydroxyl group to form N-acetyl-5-methoxytryptamine or melatonin, by Hydroxyindol-O-methyltransferase, also known as N-acetylserotonin-O-methyltransferase (17) (Fig. 1). Following its synthesis, melatonin is released into cerebrospinal fluid and circulation and the circulating melatonin has a half-life of 20-30 min, with most of its metabolism occuring in liver via cytochrome P450-mediated oxygenation (16). Melatonin biosynthesis and secretion are low during daytime and high during night-time and this pattern of the circadian rhythm is regulated by neural circuits composed of neural fibers originating from retina and passing via retino-hypothalamic tract to the SCN of the hypothalamus (18). SCN is composed of special ganglion cells containing a photo-pigment, known as melanopsin (19). During night-time, the release of norepinephrine from the postganglionic sympathetic fibers of SCN supplying to the pineal gland is elevated due to reduced electrical signal activity in SCN. Norepinephrine activates the $\beta$-adrenergic receptors present on the pinealocytes leading to the activation of adenylyl cyclase-cyclic AMP system and melatonin biosynthesis. During daytime, norepinephrine release from the postganglionic sympathetic nerve fibers is suppressed because of elevated electrical signals in the SCN, thereby lowering activation of melatonin synthesis and release in pinealocytes (20).

Many of the physiological effects of melatonin are exerted via its interaction and activation of specific receptors present on the cell membrane surface as well as the nucleus. Melatonin has at least two receptors, MT1 and MT2, belonging to the G-protein receptor family, on cell membranes, and a third MT3 receptor, which is a quinine reductase (16). Melatonin also activates nuclear receptors of melatonin belonging to the
Melatonin biosynthesis

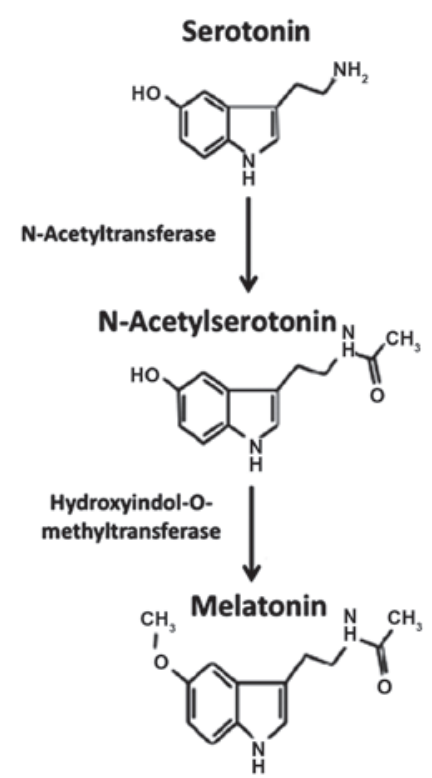

Figure 1. Biosynthesis of melatonin. Melatonin biosynthesis begins with the conversion of tryptophan to serotonin, which is acetylated by arylalkylamine- $\mathrm{N}$-acetyltransferase to form $\mathrm{N}$-acetyl-serotonin, followed by methylation at the 5-hydroxyl group to form $\mathrm{N}$-acetyl-5-methoxytryptamine or melatonin, by Hydroxyindol-O-methyltransferase, also known as $\mathrm{N}$-acetylserotonin-O-methyltransferase.

RZR/ROR orphan receptor type and include three subtypes $(\alpha, \beta, \gamma)$ and four splicing variants of the $\alpha$-subtype melatonin receptor (21). Most of the pharmacological effects of melatonin are also thought to be mediated through activation of these receptors. Besides membrane and nuclear receptors, melatonin also interacts with some cytosolic proteins and enzymes, and exerts antioxidant and other effects (22). In many tissues, MT1 receptor activation seems to mediate melatonin action through

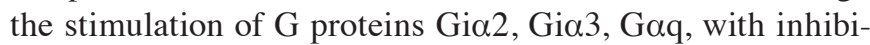
tory effects on the cAMP signaling pathways (23). By contrast, MT2 receptors, are coupled to phosphoinositide signal transduction pathways and the inhibition of adenylyl cyclase and guanylyl cyclase pathways (24) (Fig. 2).

\section{Melatonin and pain}

The precise mechanisms underlying the analgesic effects of melatonin are not known although several possibilities have been suggested, which include the involvement of $\beta$-endorphins, GABA receptor, opioid 1-receptors and the nitric oxide (NO)-arginine pathway (25). Melatonin increases the release of $\beta$-endorphin from pituitary gland, and it has been observed that naloxone, which blocks $\beta$-endorphin binding to opioid receptors, may antagonize the melatonin-induced nociceptive effects $(26,27)$. Melatonin may also mediate its analgesic activity by interacting with opioidergic, benzodiazepinergic, muscarinic, nicotinic, serotonergic, and $\alpha 1$ and $\alpha 2$-adrenergic receptors located in the central nervous system and also in the dorsal horn of the spinal cord (12). Furthermore, inasmuch as melatonin-induced long-term analgesia may be antagonized by naloxone, opioid receptors are likely involved in melatonin action (28). 


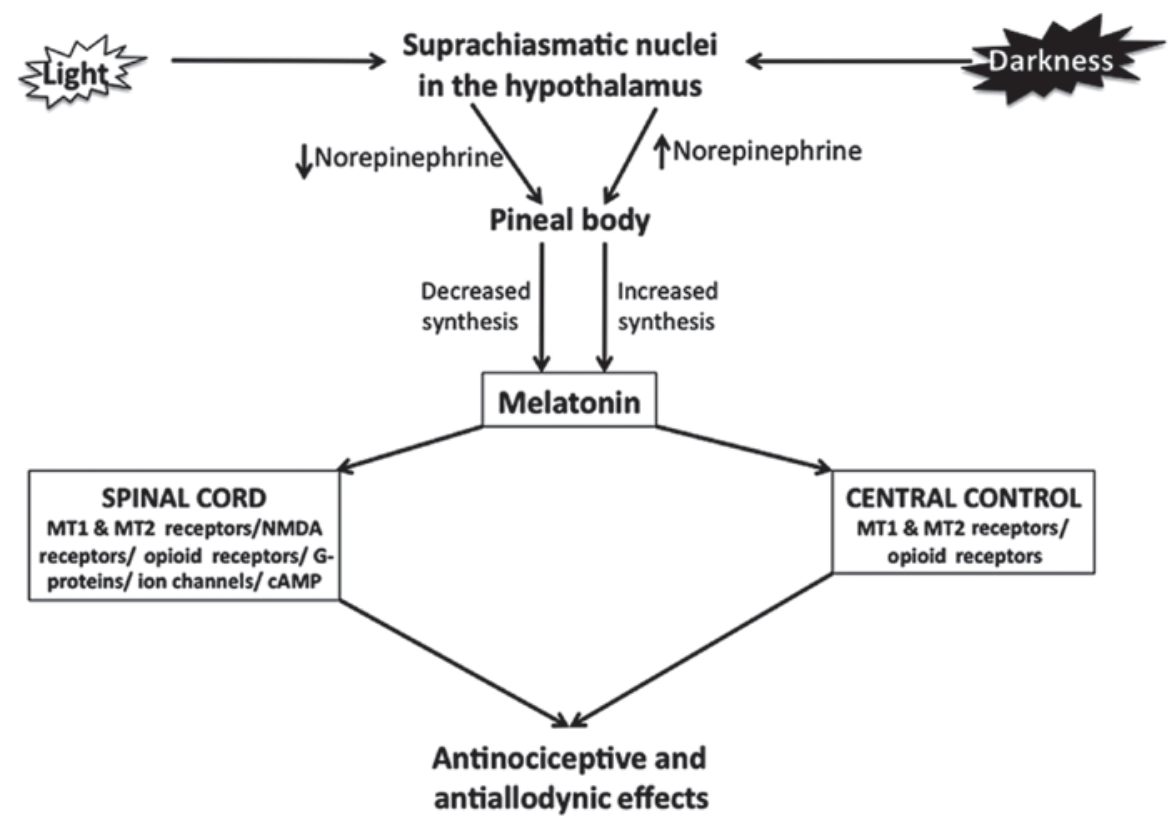

Figure 2. Control of melatonin synthesis in the pineal body by light-dark cycles and antinociceptive and antiallodynic effects of melatonin. Suprachiasmatic nucleus (SCN) in hypothalamus is composed of special ganglion cells containing a photo-pigment, known as melanopsin. During night-time, the release of norepinephrine from the postganglionic sympathetic fibers of SCN supplying to the pineal gland is elevated due to reduced electrical signal activity in SCN. Norepinephrine activates the $\beta$-adrenergic receptors present on the pinealocytes leading to melatonin biosynthesis. During daytime, norepinephrine release from the postganglionic sympathetic nerve fibers is suppressed because of elevated electrical signals in the SCN, thereby lowering the activation of melatonin synthesis and release in pinealocytes. Melatonin appears to act via its MT1/MT2 receptors in the spinal cord and in brain and by its interaction with other receptors such as opioid, substance P and NMDA.

In several conditions of chronic pain such as fibromyalgia (FM), inflammatory bowel syndrome and migraine, melatonin has been found to be effective in reducing pain (29-31). Although it has been suggested that melatonin may have analgesic and anxiolytic effects in the perioperative period, its effectiveness has been controversial (32). In several experimental animal models of pain, melatonin has been shown to be efficacious. Thus, in models of electrically induced pain, intraperitoneal (i.p.) injection of melatonin was able to increase the anti-nociceptive effect up to $210 \mathrm{~min}$ (33). Similarly, in a rodent model of thermally induced pain measured as tail-flick, melatonin ( $\leq 120 \mathrm{mg} / \mathrm{kg}$, i.p.) produced a significant analgesic response (34). In a hot-plate model of pain induction in mice, it was observed that melatonin exerted maximal analgesic effect, when administered to the mice in the evening and that these effects of melatonin could be blocked by an opiate antagonist naloxone or central benzodiazepine antagonist flurmazenil, indicating an interplay of these receptor pathways in melatonin action (35). In a paw-withdrawal test of a neuropathic pain model, administration of melatonin dose dependently exerted analgesic effects lasting for a period of $1 \mathrm{~h}$ (36). In a model of mechanically induced pain via tail clamping,2-bromomelatonin was found to induce dose-dependent analgesic effect (37). Chemically induced pain, which resembles acute pain in humans, was also reduced by melatonin (38). Melatonin was found to reduce inflammatory pain, probably by blocking the production of $\mathrm{NO}$ by inducible $\mathrm{NO}$ synthase and the signaling pathways of NO-cyclic GMP $(33,39)$. In all these different animal models of pain, administration of melatonin had no adverse effects. There are also limitations with regard to the ability of melatonin to act as an anti-nociceptive agent. Thus, in a mouse model of neuropathic pain, where the mice underwent a tight ligation of sciatic nerve, melatonin was able to reduce paw-withdrawal latencies, which is a measure of thermal hyperanalgesia, but had no significant effect on withdrawal thresholds, a measure of mechanical allodynia (36).

\section{Pharmacological use of melatonin in humans}

Strong chronobiotic and hypnotic properties and the ability to correct sleep-wake rhythm disturbances, make melatonin as a drug choice for decreasing sleep latency. It has been observed that when administered at the right time and dose, melatonin was able to effectively correct the circadian rhythms in children with sleep disorders and because of this, melatonin is commonly used in children of all ages with sleep-related issues (40-43). There are no clinical guidelines for proper prescription of melatonin in children suffering with different neurological disorders, albeit many pediatricians currently prescribe melatonin to their patients, considering this as a naturally occurring sleeping aid (44). Timing of melatonin administration is critical in achieving expected results of treatment, because of its biological rhythm-based secretion and effects. It is important to administer melatonin earlier than the dim-light melatonin onset for optimal treatment effects, as administration 2-3 $\mathrm{h}$ after this onset has no effects (45).

\section{Melatonin and migraine}

Considering that there is strong relationship between sleep and headache, the ability of melatonin to regulate sleep disorders may also be useful in improving headache pathophysiology (46). It has been reported that properly timed melatonin treatment with appropriate dosing, decreased 
headaches in $78.6 \%$ of 328 patients suffering with circadian rhythm sleep disorders and headache, while adverse effects (slight headache) were observed in $13.8 \%$ of 676 patients with only circadian rhythm sleep disorders without headache (47). Melatonin at a dose of $3 \mathrm{mg}$ twice daily, given to children with primary headache, was able to reduce the number, intensity and duration of headache attacks (48). It has been suggested that melatonin may be considered an effective prophylactic medication for use in children with migraine. Migraine, which is characterized by severe headache, neurologic dysfunction, sleep disturbances and pain-free intervals is commonly seen in women aged 10-40 years. In an open-labeled clinical trial with 34 patients suffering from migraine, prophylactic use of melatonin at $3 \mathrm{mg}$, given $30 \mathrm{~min}$ before bedtime, was found to reduce headache intensity as well as frequency and duration, with significant clinical improvement by 1 month (49). In another recent study, agomelatine, a melatonin agonist was found to be efficacious in the treatment of migraine patients in decreasing migraine attack frequency and duration (13).

\section{Melatonin and fibromyalgia}

FM is characterized by tenderness, altered sleep pattern and a number of painful trigger points. Although imbalanced melatonin secretion has been thought to contribute to the pathophysiology FM, there is no clear consensus on this as experimental findings on melatonin levels in FM patients were not consistent $(29,50,51)$. In an open-label, randomized-clinical trial that included 21 female patients, oral therapy of melatonin at doses of $3 \mathrm{mg} /$ day, $30 \mathrm{~min}$ before sleeping time, for 1 month led to significant improvement, not only in sleep quality, but also in much less painful trigger points as compared to the situation prior to treatment. Results of that study also suggested that melatonin treatment has the potential to improve pain, fatigue and symptoms of depression (52). In another double-blind and placebo-controlled study in $101 \mathrm{FM}$ patients it was observed that melatonin treatment alone had significant effects in improving pain, fatigue, rest/sleep, stiffness, comparable to a serotonin reuptake blocker, fluoxetine (53). Combination therapy with melatonin and fluoxetine showed much better reduction in the anxiety score and fatigue in these patients and also greatly decreased depression. A more recent randomized clinical study that included 63 female patients with FM tested the efficacy of 6-week treatment with melatonin versus melatonin plus amitriptyline, another serotonin reuptake inhibitor and the results showed that a combined therapy of amitriptyline and melatonin, and melatonin as a monotherapy were more efficient than amitriptyline monotherapy in improving pain, morning stiffness, and sleep disorders (54). Thus, combined melatonin plus a selective serotonin reuptake inhibitor treatment requires further large-scale clinical evaluation in FM patients.

\section{Irritable bowel syndrome and melatonin}

Irritable bowel syndrome that affects the GI tract is a painful condition characterized by abdominal pain, flatulence, constipation and diarrhea in association with sleep disturbances. GI tract is known to create and release an almost 400 -fold higher amount of melatonin than the pineal gland, but without circadian rhythms as is the case in the pineal body. Although the precise physiological function of melatonin from GI tract is not clear, food intake affects melatonin synthesis and release (55). Considering that irritable bowel syndrome affects $11-20 \%$ of the adult population (56), the possibility that GI tract melatonin may have some physiological roles in the control of this pain exists. Melatonin was found to reduce abdominal pain, aid defecation in patients with irritable bowel syndrome (57), and to exert positive effects on extracolonic symptoms via sedative/anxiolytic and/or direct effects on the GI tract itself (58). Involvement of the central nervous system in the action of melatonin in relieving pain in irritable bowel syndrome is a strong possibility, which needs further verification (57). In two separate randomized placebo-controlled clinical trials, women with irritable bowel syndrome were treated with melatonin for 4-6 weeks and in both the trials, it was observed that melatonin was able to improve the symptoms and lower the associated pain $(31,56)$.

\section{Melatonin in the control of peri-operative outcomes}

Anxiety and pain during surgical manipulation procedures are common and this is generally controlled by systemic analgesia and sedation. The common practice of using benzodiazepine preoperatively leads to a number of side effects. Inasmuch as melatonin does not have any adverse effects, and as it has anxiolytic and pain-controlling effects, it has been suggested that melatonin has beneficial effects on the peri-operative outcomes. It was concluded that while melatonin premedication can be effective in ameliorating preoperative anxiety in adults, its ability to control pain remains controversial in the perioperative period (32). Peri-operative anxiety is known to aggravate postoperative pain. A recent systematic meta-analysis study that included 24 studies with a total of 1,794 patients, suggested that melatonin reduced the preoperative anxiety score and postoperative pain score, as compared with placebo, although the authors cautioned that there was a great degree of heterogeneity among the data (59). In a double-blind placebo controlled study, pre-operative treatment of patients with melatonin significantly decreased pain and anxiety during the first $36 \mathrm{~h}$ after surgery (60). In another study on patients undergoing elective hand surgery, premedication with melatonin was able to reduce the level of anxiety and enhanced intraoperative and postoperative analgesia (61). Similar postoperative analgesic results were obtained with the use of melatonin following cataract surgery under topical anesthesia (62).

\section{Conclusions}

Melatonin is a hormone produced from tryptophan in the pineal body and is involved in the regulation of circadian and biological rhythms and the associated physiological responses such as sleep, anxiety and pain. Anti-nociceptive and antiallodynic effects of melatonin are demonstrated, not only in various animal models of pain perception, but also in patients under different pathological and surgical intervention conditions. Melatonin appears to act via its MT1/MT2 receptors at the dorsal horn levels of the spinal cord and interacts with other receptors including opioid, substance P and NMDA. 
Furthermore, melatonin actions may be antagonized by antagonists of opioid and benzodiazepine receptors. Clinical studies indicated that a combination of serotonin reuptake blockers and melatonin is more efficacious in pain and anxiety control. However, the exact mechanism remains to be examined in future studies.

\section{References}

1. Bolten WW, Gross M, Brabant T, Weck V and Labenz J: [Individual pain treatment with NSAIDs]. MMW Fortschr Med 155: 59-60, 62, 2013.

2. Ennis ZN, Dideriksen D, Vaegter HB, Handberg G and Pottegard A: Acetaminophen for Chronic Pain: A Systematic Review on Efficacy. Basic Clin Pharmacol Toxicol 118: 184-189, 2016.

3. Pasternak GW: When it comes to opiates, just say NO. J Clin Invest 117: 3185-3187, 2007.

4. García Rodríguez LA and Jick H: Risk of upper gastrointestinal bleeding and perforation associated with individual non-steroidal anti-inflammatory drugs. Lancet 343: 769-772, 1994.

5. Wiech K and Tracey I: The influence of negative emotions on pain: Behavioral effects and neural mechanisms. Neuroimage 47 987-994, 2009

6. Basbaum AI, Bautista DM, Scherrer G and Julius D: Cellular and molecular mechanisms of pain. Cell 139: 267-284, 2009.

7. Tovote P, Fadok JP and Lüthi A: Neuronal circuits for fear and anxiety. Nat Rev Neurosci 16: 317-331, 2015.

8. Wilhelmsen M, Amirian I, Reiter RJ, Rosenberg J and Gögenur I: Analgesic effects of melatonin: A review of current evidence from experimental and clinical studies. J Pineal Res 51: 270-277, 2011.

9. Frange C, Hachul H, Tufik S and Andersen ML: Circadian rhythms, insomnia and osteoarthritis pain. Chronobiol Int 32: $1323-1324,2015$.

10. Junker U and Wirz S: Review article: Chronobiology: Influence of circadian rhythms on the therapy of severe pain. J Oncol Pharm Pract 16: 81-87, 2010.

11. Lucas RJ, Lall GS, Allen AE and Brown TM: How rod, cone, and melanopsin photoreceptors come together to enlighten the mammalian circadian clock. Prog Brain Res 199: 1-18, 2012.

12. Srinivasan V, Lauterbach EC, Ho KY, Acuña-Castroviejo D, Zakaria R and Brzezinski A: Melatonin in antinociception: Its therapeutic applications. Curr Neuropharmacol 10: 167-178, 2012.

13. Danilov A and Kurganova J: Melatonin in chronic pain syndromes. Pain Ther 5: 1-17, 2016.

14. Reiter RJ: Melatonin: The chemical expression of darkness. Mol Cell Endocrinol 79: C153-C158, 1991.

15. Pickard GE: Circadian rhythm of nociception in the golden hamster. Brain Res 425: 395-400, 1987.

16. Srinivasan V, Pandi-Perumal SR, Spence DW, Moscovitch A, Trakht I, Brown GM and Cardinali DP: Potential use of melatonergic drugs in analgesia: Mechanisms of action. Brain Res Bull 81: 362-371, 2010

17. Axelrod $\mathrm{J}$ and Wurtman RJ: Photic and neural control of indoleamine metabolism in the rat pineal gland. Adv Pharmacol 6 (Pt A): 157-166, 1968.

18. Moore RY: Neural control of the pineal gland. Behav Brain Res 73: 125-130, 1996.

19. Berson DM, Dunn FA and Takao M: Phototransduction by retinal ganglion cells that set the circadian clock. Science 295 1070-1073, 2002.

20. Klein DC, Schaad NL, Namboordiri MA, Yu L and Weller JL: Regulation of pineal serotonin $\mathrm{N}$-acetyltransferase activity. Biochem Soc Trans 20: 299-304, 1992.

21. Becker-André M, Wiesenberg I, Schaeren-Wiemers N, André E, Missbach M, Saurat JH and Carlberg C: Pineal gland hormone melatonin binds and activates an orphan of the nuclear receptor superfamily. J Biol Chem 269: 28531-28534, 1994.

22. Mukherjee D, Roy SG, Bandyopadhyay A, Chattopadhyay A, Basu A, Mitra E, Ghosh AK, Reiter RJ and Bandyopadhyay D Melatonin protects against isoproterenol-induced myocardial injury in the rat: Antioxidative mechanisms. J Pineal Res 48: 251-262, 2010

23. Brydon L, Roka F, Petit L, de Coppet P, Tissot M, Barrett P, Morgan PJ, Nanoff C, Strosberg AD and Jockers R: Dual signaling of human Mel1a melatonin receptors via G(i2), G(i3), and G(q/11) proteins. Mol Endocrinol 13: 2025-2038, 1999.
24. Boutin JA, Audinot V, Ferry G and Delagrange P: Molecular tools to study melatonin pathways and actions. Trends Pharmacol Sci 26: 412-419, 2005.

25. Marseglia L, D'Angelo G, Manti S, Aversa S, Arrigo T, Reiter RJ and Gitto E: Analgesic, anxiolytic and anaesthetic effects of melatonin: New potential uses in pediatrics. Int J Mol Sci 16: 1209-1220, 2015

26. Yu CX, Zhu B, Xu SF, Cao XD and Wu GC: The analgesic effects of peripheral and central administration of melatonin in rats. Eur J Pharmacol 403: 49-53, 2000.

27. Shavali S, Ho B, Govitrapong P, Sawlom S, Ajjimaporn A, Klongpanichapak S and Ebadi M: Melatonin exerts its analgesic actions not by binding to opioid receptor subtypes but by increasing the release of beta-endorphin an endogenous opioid. Brain Res Bull 64: 471-479, 2005.

28. Lakin ML, Miller CH, Stott ML and Winters WD: Involvement of the pineal gland and melatonin in murine analgesia. Life Sci 29: 2543-2551, 1981

29. Mease P: Fibromyalgia syndrome: Review of clinical presentation, pathogenesis, outcome measures, and treatment. J Rheumatol Suppl 75: 6-21, 2005.

30. Reiter RJ, Acuna-Castroviejo D and Tan DX: Melatonin therapy in fibromyalgia. Curr Pain Headache Rep 11: 339-342, 2007.

31. Song GH, Leng PH, Gwee KA, Moochhala SM and Ho KY: Melatonin improves abdominal pain in irritable bowel syndrome patients who have sleep disturbances: A randomised, double blind, placebo controlled study. Gut 54: 1402-1407, 2005.

32. Yousaf F, Seet E, Venkatraghavan L, Abrishami A and Chung F: Efficacy and safety of melatonin as an anxiolytic and analgesic in the perioperative period: A qualitative systematic review of randomized trials. Anesthesiology 113: 968-976, 2010

33. El-Shenawy SM, Abdel-Salam OM, Baiuomy AR, El-Batran S and Arbid MS: Studies on the anti-inflammatory and anti-nociceptive effects of melatonin in the rat. Pharmacol Res 46: 235-243, 2002

34. Wang T, Li SR, Dai X, Peng YL, Chen Q and Wang R: Effects of melatonin on orphanin $\mathrm{FQ} /$ nociceptin-induced hyperalgesia in mice. Brain Res 1085: 43-48, 2006.

35. Golombek DA, Escolar E, Burin LJ, De Brito Sánchez MG and Cardinali DP: Time-dependent melatonin analgesia in mice: Inhibition by opiate or benzodiazepine antagonism. Eur J Pharmacol 194: 25-30, 1991.

36. Ulugol A, Dokmeci D, Guray G, Sapolyo N, Ozyigit F and Tamer M: Antihyperalgesic, but not antiallodynic, effect of melatonin in nerve-injured neuropathic mice: Possible involvements of the L-arginine-NO pathway and opioid system. Life Sci 78: 1592-1597, 2006.

37. Naguib M, Baker MT, Spadoni G and Gregerson M: The hypnotic and analgesic effects of 2-bromomelatonin. Anesth Analg 97: 763-768, 2003

38. Mantovani M, Kaster MP, Pertile R, Calixto JB, Rodrigues AL and Santos AR: Mechanisms involved in the antinociception caused by melatonin in mice. J Pineal Res 41: 382-389, 2006

39. Hernández-Pacheco A, Araiza-Saldaña CI, Granados-Soto V and Mixcoatl-Zecuatl T: Possible participation of the nitric oxide-cyclic GMP-protein kinase G-K+ channels pathway in the peripheral antinociception of melatonin. Eur J Pharmacol 596: 70-76, 2008.

40. van Geijlswijk IM, Korzilius HP and Smits MG: The use of exogenous melatonin in delayed sleep phase disorder: A meta-analysis. Sleep 33: 1605-1614, 2010.

41. Braam W, van Geijlswijk I, Keijzer H, Smits MG, Didden R and Curfs LM: Loss of response to melatonin treatment is associated with slow melatonin metabolism. J Intellect Disabil Res 54: 547-555, 2010.

42. Appleton RE and Gringras P: Melatonin: Helping to MEND impaired sleep. Arch Dis Child 98: 216-217, 2013.

43. Hartz I, Furu K, Bratlid T, Handal M and Skurtveit S: Hypnotic drug use among 0-17 year olds during 2004-2011: A nationwide prescription database study. Scand J Public Health 40: 704-711, 2012.

44. Heussler H, Chan P, Price AM, Waters K, Davey MJ and Hiscock H: Pharmacological and non-pharmacological management of sleep disturbance in children: An Australian Paediatric Research Network survey. Sleep Med 14: 189-194, 2013

45. Burgess HJ, Revell VL, Molina TA and Eastman CI: Human phase response curves to three days of daily melatonin: $0.5 \mathrm{mg}$ versus $3.0 \mathrm{mg}$. J Clin Endocrinol Metab 95: 3325-3331, 2010.

46. Reiter RJ, Tan DX and Galano A: Melatonin: Exceeding expectations. Physiology (Bethesda) 29: 325-333, 2014. 
47. Rovers J, Smits M and Duffy JF: Headache and sleep: Also assess circadian rhythm sleep disorders. Headache 54: 175-177, 2014.

48. Miano S, Parisi P, Pelliccia A, Luchetti A, Paolino MC and Villa MP: Melatonin to prevent migraine or tension-type headache in children. Neurol Sci 29: 285-287, 2008.

49. Peres MF, Zukerman E, da Cunha Tanuri F, Moreira FR and Cipolla-Neto J: Melatonin, $3 \mathrm{mg}$, is effective for migraine prevention. Neurology 63: 757, 2004.

50. Klerman EB, Goldenberg DL, Brown EN, Maliszewski AM and Adler GK: Circadian rhythms of women with fibromyalgia. J Clin Endocrinol Metab 86: 1034-1039, 2001.

51. Wikner J, Hirsch U, Wetterberg L and Röjdmark S Fibromyalgia - a syndrome associated with decreased nocturnal melatonin secretion. Clin Endocrinol (Oxf) 49: 179-183, 1998.

52. Citera G, Arias MA, Maldonado-Cocco JA, Lázaro MA, Rosemffet MG, Brusco LI, Scheines EJ and Cardinalli DP: The effect of melatonin in patients with fibromyalgia: A pilot study. Clin Rheumatol 19: 9-13, 2000.

53. Hussain SA, Al-Khalifa II, Jasim NA and Gorial FI: Adjuvant use of melatonin for treatment of fibromyalgia. J Pineal Res 50 267-271, 2011.

54. de Zanette SA, Vercelino R, Laste G, Rozisky JR, Schwertner A, Machado CB, Xavier F, de Souza IC, Deitos A, Torres IL, et al: Melatonin analgesia is associated with improvement of the descending endogenous pain-modulating system in fibromyalgia: A phase II, randomized, double-dummy, controlled trial. BMC Pharmacol Toxicol 15: 40, 2014.

55. Konturek SJ, Konturek PC, Brzozowski T and Bubenik GA: Role of melatonin in upper gastrointestinal tract. J Physio Pharmacol 58 (Suppl 6): 23-52, 2007.
56. Lu WZ, Gwee KA, Moochhalla S and Ho KY: Melatonin improves bowel symptoms in female patients with irritable bowel syndrome: A double-blind placebo-controlled study. Aliment Pharmacol Ther 22: 927-934, 2005.

57. Mozaffari S, Rahimi R and Abdollahi M: Implications of melatonin therapy in irritable bowel syndrome: A systematic review. Curr Pharm Des 16: 3646-3655, 2010.

58. Saha L, Malhotra S, Rana S, Bhasin D and Pandhi P: A preliminary study of melatonin in irritable bowel syndrome. J Clin Gastroenterol 41: 29-32, 2007.

59. Andersen LP, Werner MU, Rosenberg J and Gögenur I: A systematic review of peri-operative melatonin. Anaesthesia 69: 1163-1171, 2014.

60. Caumo W, Torres F, Moreira NL Jr, Auzani JA, Monteiro CA, Londero G, Ribeiro DF and Hidalgo MP: The clinical impact of preoperative melatonin on postoperative outcomes in patients undergoing abdominal hysterectomy. Anesth Analg 105: 1263-1271, 2007.

61. Mowafi HA and Ismail SA: Melatonin improves tourniquet tolerance and enhances postoperative analgesia in patients receiving intravenous regional anesthesia. Anesth Analg 107: 1422-1426, 2008

62. Ismail SA and Mowafi HA: Melatonin provides anxiolysis, enhances analgesia, decreases intraocular pressure, and promotes better operating conditions during cataract surgery under topical anesthesia. Anesth Analg 108: 1146-1151, 2009. 